\title{
Characterization and reproducibility of canoe slalom simulated races: physiological, technical and performance analysis
}

\author{
NATHÁLIA ARNOSTI VIEIRA - LEONARDO HENRIQUE DALCHECO MESSIAS, MARCELO VOLLET \\ CARDOSO, HOMERO GUSTAVO FERRARI, SÉRGIO AUGUSTO CUNHA, DENIS ROBERTO \\ TEREZANI, FÚLVIA BARROS MANCHADO-GOBATTO
}

Laboratory of Physiology Apllied to Sport, University of Campinas, São Paulo, Brasil

\begin{abstract}
Vieira, N.A., Mesias, L.H., Cardoso, M.V., Ferrari, H.G., Cunha, S.A., Terezani, D.R., \& Manchado-Gobatto, F.B. (2015). Characterization and reproducibility of canoe slalom simulated races: physiological, technical and performance analysis. J. Hum. Sport Exerc., 10(4), pp.835-846. The aims of this study were to characterise and test the reproducibility of canoe slalom race simulations performed on two different days, analysing the physiological, technical and performance responses characteristic of the sport. Six high-performance male canoe slalom K1


twelve gates. Each simulation was analysed the runtime, distance travelled, mean velocity; images were captured by the digital camera (JVC) and determined quantitative variables. Heart monitors (Polar, RS800x model) were used to record heart rate during race simulations and recovery; data were stored every $5 \mathrm{~s}$, to determine the blood lactate concentration ([Lac]), blood samples were collected from the earlobe at rest and after 1, 3, 5, 7 and 9 min of race simulation (recovery). Tests of normality (Shapiro Wilk) and variance (Levene) were applied. The variables were compared using paired t-tests. Intraclass coefficient correlation (ICC) and Pearson product moment were used for relationship. In all cases, the level of significance was pre-fixed at $5 \%$. Differences between the first and second simulations could not be noticed. Another important indicator was the high correlation found between the runtime $(I C C=0.71)$, distance travelled $(I C C=0.77)$, mean velocity $(I C C=0.80)$ and total number of paddles $(I C C=0.79)$. The lactate levels on minutes $3,5,7$ and 9 also pointed towards strong correlations (ICC=0.88, ICC $=0.90$, ICC $=0.95$ and ICC $=0.90$ respectively), which may indicate that the adopted simulation model seems to be practicable and of great value to canoe slalom evaluations. Keywords: SIMULATED RACE CHARACTERIZATION, PERFORMANCE, REPRODUCIBILITY, CANOE SLALOM.
\end{abstract}

\footnotetext{
Corresponding author. University of Campinas - Unicamp, Campinas, SP

E-mail: nathaliaarnosti@gmail.com

Submitted for publication May 2014

Accepted for publication January 2014

JOURNAL OF HUMAN SPORT \& EXERCISE ISSN 1988-5202

(C) Faculty of Education. University of Alicante

doi:10.14198/jhse.2015.104.01
} 


\section{INTRODUCTION}

Among aquatic Olympic sports, it is possible to highlight canoe slalom. Competitions are held on a water course with different degrees of difficulty with the designated path outlined by "gates" (beacons). This sport also features extrinsic and natural factors, such as water depth, wave magnitude, presence of natural setbacks, and finally, current speed (Messias, Reis, Ferrari \& Manchado-Gobatto, 2014; Shephard, 1987). Depending on the course and class of vessel used, the duration of official canoe slalom competitions ranges from 90 to $120 \mathrm{~s}$ (Nibali, Hopkins \& Drinkwater, 2011), with the objective of completing the course in the shortest time possible without committing any faults (Michael, Smith \& Rooney, 2010).

In this sense, accurate and fast completion of the course depends on a series of technical movements executed at a high speed with changes in direction. These movements are necessarily linked to the successful development of basic physical capacities skills, such as aerobic and anaerobic resistance, strength, coordination, and speed, all of which are required during competition (Zamparo et al., 2006). Notwithstanding, understanding about the particularities of a sport depends on characterisation of the competitive moments. In this manner, quantitative and qualitative analysis of the physical, technical, tactical and physiological aspects allows for more specific interventions during the training process (Matveev, 1997).

Simulating competition allows the assessment of performance in accordance with the specific regulations of the sport and provides direct contact with competitors, encouraging athletes to develop the necessary motivation to achieve victory. Several assessments are used during race simulations, such as filming (Carling, Bloomfield, Nelsen \& Reilly, 2008; Davies, 2003; Hunter, Cochrane \& Sachlikidis, 2007, 2008) and monitoring physiological variables (Gray \& Jenkins, 2010; Hill-Haas, Dawson, Coutts \& Rowsell, 2009; Vieira et al., 2011; Vieira et al., 2010), in an attempt to predict physiological and psychological conditions during competition. Additionally, in non-televised sporting events, such as slalom kayaking, this feature is even more significant, providing quantitative and qualitative information to coaches and athletes (Hughes \& Bartlett, 2002; Hunter et al., 2007, 2008) and offering a non-invasive method of analysing performance during competition (Bloomfield, Jonsson, Polman, Houlahan \& O'Donoghue, 2005).

Valid and reproducible quantitative analysis should complement subjective judgments of performance (Schokman, Le Rossignol \& Sparrow, 2002). Specifically reproducibility refers to the stability of the measure when a test protocol is executed repeatedly (Hopkins, Schabort \& Hawley, 2001). Knowing that a measurement is reproducible is important because a lack of reproducibility can lead to high measurement error, potentially leading to inaccurate data or an inability to detect differences between individuals and/or observed alterations. For canoe slalom, race simulations are used widely despite the presence of extrinsic and natural variability. This study was conducted despite the limited number of studies seeking to comprehend this sport and the reproducibility of simulations used to evaluate it. Although race simulations are used widely in canoe slalom, few studies have sought to comprehend the impact of extrinsic and natural variability on the reproducibility of these assessments.

In this sense, the aims of this study were to characterise and test the reproducibility of canoe slalom race simulations performed on two different days, analysing the physiological, technical and performance responses characteristic of the sport. 


\section{MATERIAL AND METHODS}

\section{Participants}

Six high-performance male canoe slalom K1 athletes (age 17 \pm 2 years; body mass; $68 \pm 5 \mathrm{~kg}$; and height $175 \pm 6 \mathrm{~cm}$ ) underwent two race simulations with an interval of 72 hours between them. The athletes included in this study are regular participants in national and international competitions, with five of these athletes ranked among the top eight in the Brazilian Canoe Confederation national rankings. Three of the athletes achieved a ranking sufficient to join the Brazilian Canoe slalom Team next year. All participants were informed of the procedures, requirements, risks and benefits before signing the consent form. The experimental procedures were approved by the Ethics Committee in Research of the institution where the study was developed.

\section{Experimental design}

Race simulations were performed on two different days in the usual environment of the athletes' training and competitions. The artificial course was assembled on both days and consisted of twelve "gates", characterised by two rods separated by a space of $1.2-4.0 \mathrm{~m}$. Eight of the gates were aligned such that the kayak would pass through with the current while the other four were aligned such that the kayak would pass through against the current (where the bow of the kayak is pointed toward the river's nascent). There was an interval of 72 hours between the two race simulations with similar weather conditions on both days. On both occasions, the river flow was $65.74 \mathrm{~m}^{3} / \mathrm{s}$, air temperature was between $23.4^{\circ} \mathrm{C}$ and $24.4^{\circ} \mathrm{C}$, and the water temperature was $16 \pm 1^{\circ} \mathrm{C}$. This information was provided by the Municipal Water Service and Sewer (SEMAE) of the city of Piracicaba, SP, Brazil.

As described later, each simulation was recorded and analysed according to performance parameters (runtime, distance travelled, mean velocity), technical gestures executed by the kayakers (quantitative analysis of the total number of paddles and the number of paddles with and against the current) and physiological responses such heart rate (HR) throughout the race and lactate concentration ([Lac]) before and after the race.

The necessary time to complete the course is used as the definitive criterion of performance in canoe slalom (Michael, Rooney \& Smith, 2008). As runtime is such a crucial variable, it was determined using a stopwatch (Casio, HS-30W-N1V) and a digital camera (JVC) positioned on the side of the river, approximately in the middle of the path, which allowed for a full view of the course. The timing equipment was triggered by athletes moving toward the course and finished after passage through the final gate. A global positioning system (GPS-G3 model, Polar) was used to quantify the distance travelled by each athlete as well as the trajectory during the race simulations. Mean velocity $\left(\mathrm{km}^{\mathrm{n}} / \mathrm{h}^{-1}\right)$ for each simulation was calculated using the measurements of distance travelled $(\mathrm{m})$ and runtime $(\mathrm{s})$ obtained by the GPS.

\section{Technical executions}

After images were captured by the digital camera (JVC), two evaluators reviewed the videos using visual aids and determined quantitative variables that are significant for slalom kayaking competitions. The analysed variables were as follows: total number of paddles (Total.Paddles), interpreted as each cycle that the oar went into and out of the water; paddling with the current (Pad.with), which occurs when paddling is with the current; paddling against the current (Pad.Against), defined by the position of the bow pointing to the nascent of the river; complete cycle of paddling (Compl.cycle), characterised by each cycle defined as the alternating right/left paddle; and finally, cross paddling (Cross.Paddling), which occurred when the athlete used the same upper limb two or more times consecutively to perform subsequent paddling. No 
biomechanical, qualitative, or specific paddling technique analyses were conducted; only quantitative analysis of the kayakers' movements was performed.

\section{Heart rate}

Heart monitors (Polar, RS800x model) were used to record heart rate during race simulations and recovery; data were stored every $5 \mathrm{~s}$ and recordings were transferred to a microcomputer using a specific Polar interface. Minimum HR (Min.HR) was considered the minimum value registered; mean HR (Mean.HR) was considered as the mean of total heart rate registered during the simulation; and peak HR (Peak.HR) was considered the peak value registered. All data were calculated from the results for each of the two race simulations.

\section{Blood samples and determination of blood lactate concentration}

Blood samples were collected from the earlobe at rest and after 1, 3, 5, 7 and 9 min of race simulation (recovery). Using the lactate concentration data, it was possible to identify the peak lactate concentration, interpreted as the highest value in the sample, as well as the time corresponding to that peak.

To analyse blood lactate concentration enzymatically (Engel \& Jones, 1978), samples were stored at a temperature of $-300 \mathrm{C}$, shaken in a magnetic stirrer and centrifuged $(3000 \mathrm{rpm})$. An aliquot of $100 \mu \mathrm{L}$, equivalent to $500 \mu \mathrm{L}$ of reagent, was extracted. The homogenate was again mixed and then immediately incubated for 20 minutes at $37^{\circ} \mathrm{C}$. Samples were read in a spectrophotometer with a wavelength of 340 $\mathrm{nm}$.

\section{Statistical analyses}

The STATISTICA statistical package (version 8.0) was used to process and report the data. Tests of normality (Shapiro Wilk) and homogeneity (Levene) were applied to identify the data characteristics. As the data exhibited normality and equal variance, parametric statistical methods were adopted. The physiological, technical and performance parameters were compared using paired t-tests, with the aim to identify possible significant differences between the two race simulations. Intraclass correlation coefficients (ICC), Pearson product moment, confidence intervals (Cl) and coefficient of variation (CV) were employed for relationship analyses. The results are expressed mean \pm standard error of the mean. In all cases, the level of significance was pre-fixed at $5 \%$.

\section{RESULTS}

Table 1 displays performance results (runtime, distance travelled and mean velocity) for both simulations. Similarity was observed between the runtime of the first $(104.48 \pm 3.60 \mathrm{~s})$ and second race simulations $(103.29 \pm 3.65 \mathrm{~s})$, with a positive significant correlation and a low coefficient of variation (ICC $=0.83$; $\mathrm{CV}=4.57)$. The distance travelled in the two race simulations was not different, but it was detected, although not significant, a low and inverse correlation (ICC=-0.54). On the other hand, the estimates of mean velocity in both runs were equal and significantly correlated $(I C C=0.80)$. 
Table 1.Reproducibility of performance results such runtime (s), distance travelled $(\mathrm{m})$ andmean velocity $\left(\mathrm{km} / \mathrm{h}^{-1}\right)$ for each simulation performed by t-test (P), intraclass coefficient correlation (ICC) and coefficient of variation (CV).

\begin{tabular}{|c|c|c|c|c|c|}
\hline Performance results & $\begin{array}{c}\text { First } \\
\text { Race Simulation }\end{array}$ & $\begin{array}{c}\text { Second } \\
\text { Race Simulation }\end{array}$ & $\mathrm{P}$ & ICC & $\mathrm{CV}$ \\
\hline Runtime (s) & $104.48 \pm 3.60$ & $103.29 \pm 3.65$ & 0.68 & $0.83 *$ & 4.57 \\
\hline $\mathrm{CI}(\alpha=0.05)$ & $(2.25-8.83)^{\sharp}$ & $(2.28-8.95)^{\#}$ & $(-10.00-14.10)$ & $(0.06-0.98)$ & $(2.85-11.21)$ \\
\hline Distance travelled (m) & $208.25 \pm 11.19$ & $216.00 \pm 4.75$ & 0.77 & -0.54 & 16.82 \\
\hline $\mathrm{CI}(\alpha=0.05)$ & $(6.98-27.44)^{\#}$ & $(2.96-11.65)^{\#}$ & $(-15.00-19.10)$ & $(-0.48-0.94)$ & $(10.50-41.25)$ \\
\hline Mean velocity $\left(\mathrm{km} / \mathrm{h}^{-1}\right)$ & $7.29 \pm 0.54$ & $7.57 \pm 0.29$ & 0.78 & $0.80 *$ & 5.86 \\
\hline $\mathrm{CI}(\alpha=0.05)$ & $(0.34-1.32)^{\#}$ & $(0.18-0.71)^{\#}$ & $(-15.80-19.90)$ & $(0.03-0.98)$ & $(3.66-14.37)$ \\
\hline
\end{tabular}

Results expressed as the mean \pm standard error of the mean

*Significant correlation $(\mathrm{P} \leq 0.05)$

\#Confidence interval for standard error of the mean

Technical parameters such Total.Paddles, Pad.with, Pad.Against, Compl.cycle and Cross.Paddling for both simulations are shown in Table 2. As for the performance results, similar results were also obtained for the quantitative paddling parameters; however, the only significant correlation observed between the two simulations for the technical parameters was for the total number of paddles (ICC=0.81).

Table 2.Reproducibility of technical parameters such total number of paddles (Total.paddles), paddling with the current (Pad.With),

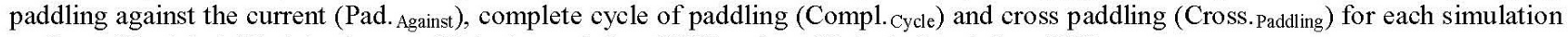
performed by t-test (P), intraclass coefficient correlation (ICC) and coefficient of variation (CV).

\begin{tabular}{|c|c|c|c|c|c|}
\hline $\begin{array}{c}\text { Technical } \\
\text { Parameters }\end{array}$ & $\begin{array}{c}\text { First } \\
\text { Race Simulation }\end{array}$ & $\begin{array}{c}\text { Second } \\
\text { Race Simulation }\end{array}$ & $\mathrm{P}$ & ICC & $\mathrm{CV}$ \\
\hline Total. Paddles $(\mathbf{n})$ & $135 \pm 3$ & $139 \pm 6$ & 0.37 & $0.81^{*}$ & 4.53 \\
\hline $\mathrm{CI}(\alpha=0.05)$ & $(1.87-7.36)$ & $(3.75-14.42)$ & $(-3.20-7.30)$ & $(0.00-0.98)$ & $(2.83-11.11)$ \\
\hline Pad. with(n) & $121 \pm 2$ & $125 \pm 5$ & 0.46 & 0.58 & 5.92 \\
\hline $\mathrm{CI}(\alpha=0.05)$ & $(1.25-4.91)$ & $(3.12-12.26)$ & $(-4.40-8.50)$ & $(-0.44-0.95)$ & $(3.70-14.52)$ \\
\hline Pad. Against (n) $_{\text {A }}$ & $14 \pm 2$ & $14 \pm 1$ & 0.93 & 0.47 & 21.89 \\
\hline $\mathrm{CI}(\alpha=0.05)$ & $(1.20-4.71)$ & $(0.62-2.45)$ & $(-53.90-58.00)$ & $(-0.55-0.93)$ & $(13.66-53.69)$ \\
\hline Compl. Cycle $(\mathbf{n})$ & $60 \pm 1$ & $63 \pm 2$ & 0.18 & 0.62 & 5.49 \\
\hline $\mathrm{CI}(\alpha=0.05)$ & $(0.77-3.02)$ & $(1.47-5.79)$ & $(-1.30-5.30)$ & $(-0.39-0.95)$ & $(3.43-13.46)$ \\
\hline Cross.Paddling (n) & $15 \pm 2$ & $12 \pm 2$ & 0.29 & 0.41 & 29.37 \\
\hline $\mathrm{CI}(\alpha=0.05)$ & $(1.24-4.86)$ & $(1.08-4.24)$ & $(-2.40-6.40)$ & $(-0.60-0.92)$ & $(18.33-72.03)$ \\
\hline
\end{tabular}

Results expressed as the mean \pm standard error of the mean

*Significant correlation $(\mathrm{P} \leq 0.05)$

${ }^{\#}$ Confidence interval for standard error of the mean

(n) $=$ number

Summary lactate concentrations at rest and after each race simulation (minutes 1, 3, 5,7 and 9) are presented in Figure 1. High values of lactate concentration were observed after race simulations, ranging

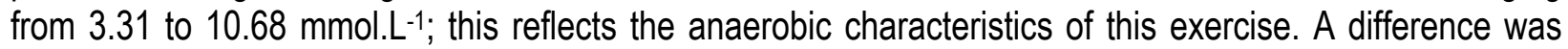
only detected between the lactate concentration at the first minute of recovery, when comparing the first 
$\left(5.61 \pm 0.52 \mathrm{mmol} . \mathrm{L}^{-1}\right)$ and second race simulations $\left(7.11 \pm 0.63 \mathrm{mmol}^{-\mathrm{L}^{-1}}\right)$. The lactate peak occurred at 5 min during the first race, while it occurred at $3 \mathrm{~min}$ in the second race; however, no differences were found between the peak lactate concentrations $\left(6.90 \pm 0.97 \mathrm{mmol}^{-L^{-1}}\right.$ and $7.50 \pm 0.50 \mathrm{mmol}^{-\mathrm{L}^{-1}}$ in the first and second races, respectively). Additionally, for all times, there were significant high correlations and low coefficient of variation between lactate concentrations at rest $(\mathrm{ICC}=0.91 ; \mathrm{CV}=5.92)$ and at minutes 3 (ICC=0.90; CV=11.77), 5 (ICC=0.93; CV=10.34), 7 (ICC=0.97; CV= 7.36) and 9 (ICC=0.95; CV=12.95).



Figure 1. Blood lactate concentration $\left(\mathrm{mmol} \cdot \mathrm{L}^{-1}\right)$ before (at rest) and after first and second race simulation (minutes $1,3,5,7$, and 9 after race).

*Difference compared to 2 st simulation at 1 minute $(\mathrm{P} \leq 0.05)$.

\#Difference compared to all other times either from 1 st as well as 2 st simulation $(\mathrm{P} \leq 0.05)$.

In the first race simulation, there was a significant correlation between the total number of paddles and [Lac] at minutes $3(r=-0.91), 5(r=-0.88), 7(r=-0.88)$ and $9(r=-0.89)$. That is, the fewer number of paddles performed by the athletes, the higher the values of [Lac]. This may be a marker of race intensity, as there was a significant correlation between runtime and total number of paddles in the second race simulation $(r=0.87)$. This indicates that a higher number of paddles during a race are correlated with a longer runtime.

The minimum, average and peak HR during the race simulations are shown in Figure 2. High HR values reveal that the race simulations were performed at a high intensity, with Mean.HR values approaching Peak.HR values. No differences were observed in HR between the two race simulations. There were positive, though not significant, correlations between the first and second race simulations (ICC $=0.20$, ICC $=0.65$ and ICC $=0.79$, for Peak. HR, Mean. HR and Min.HR, respectively). 
$\square$ 1st Simulation

2st Simulation

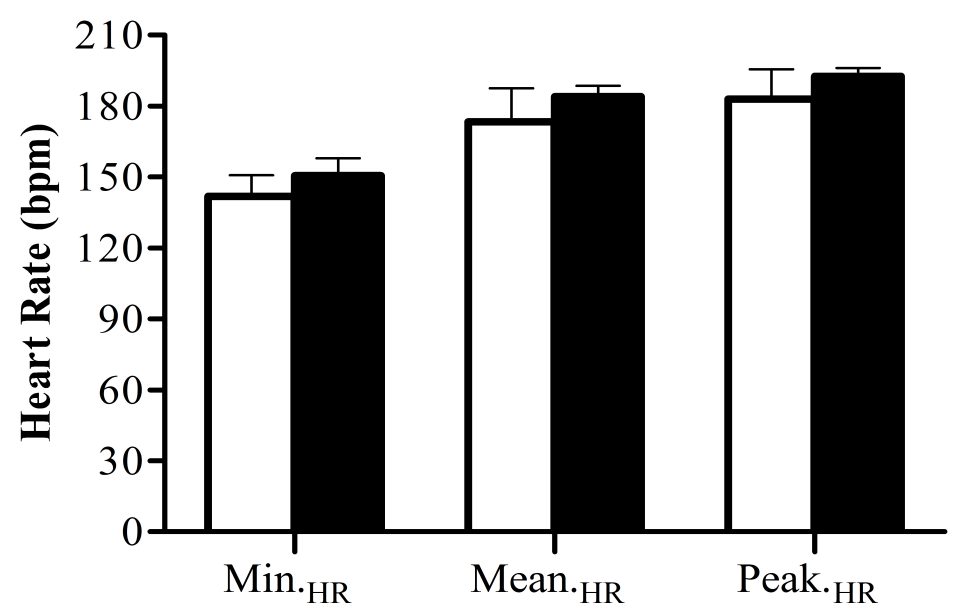

Figure 2. Minimum heart rate (Min.HR), mean heart rate (Mean.HR $)$ and peak heart rate (Peak.HR) for the two race simulations.

A significant finding concerning the intensity of this race is the correlation between mean velocity and Mean.HR in the second race simulation $(r=0.88)$. As the mean velocity is positively correlated with Mean.HR, values of Mean.HR approaching Peak.HR may reflect high mean velocity and thus, high intensity.

\section{DISCUSSION}

The main objective of a race simulation is to prepare an athlete for the specific structure of competition, providing an experience that is close to actual competition and familiarising the athlete with the performance and tactical conditions of competition (Martin, Carl \& Lehnertz, 2008). Accordingly, simulated competitions assist in diagnostic procedures of performance and provide information which can be used to analyse an athlete's progress during the training period. For this, there is a need to ascertain if the race simulations reflect actual competition characteristics and if the race simulations are reproducible.

Race simulations are widely used in canoe slalom training; however, there were no studies in the literature that evaluated the characteristics and reproducibility of these sessions. Additionally, there are many doubts and questions about the effectiveness of race simulations for this sport. This study characterised the physiological, technical and performance aspects of canoe slalom races simulation. The reproducibility of these simulations was also tested. Race simulations were conducted 72 hours apart. Both simulations were completed within the time expected for canoe slalom competitions (between 90 and $120 \mathrm{~s}$ ) with observed runtimes relatively close to those reported by Hunter et al., (2008) who analysed the semi-finals and finals of the 2005 World Championship. These authors indicated that the average runtime in the championship races was $97.9 \pm 1.3 \mathrm{~s}$. In this study, mean runtime was close to $100 \mathrm{~s}(104.5 \pm 8.8 \mathrm{~s}$ for the first and $103.3 \pm 8.9 \mathrm{~s}$ for the second simulation); there was a significant positive correlation for this parameter $(\mathrm{ICC}=0.83$ ). Different levels of inter-individual variation in runtime reveal a more homogeneous group for the 2005 World Championship (Hunter et al., 2008) compared to our sample. 
It is possible to suggest that the quality of the sample and similar extrinsic factors faced by the kayakers led to lower inter-individual variation. Zamparo et al., (2006) also investigated the runtime of athletes with medium to high performance belonging to the Italian National White Water Team. They obtained lower runtimes $(85.7 \pm 5.3 \mathrm{~s})$ but higher inter-individual variation, as was observed in this study.

The athletes in this study had mean official competition runtimes of $95.92 \pm 2.82 \mathrm{~s}$ for the first run and $97.71 \pm 3.67 \mathrm{~s}$ for the second run; these values are similar to those reported by the aforementioned studies (Nibali et al., 2011). There were significant correlations between the first run of official competitions and the first race simulation ( $r=0.94$ ), for the second official competition and the first simulated race $(r=0.85)$ and for the second official competition and the second simulated race $(r=0.88)$. In another official competition in which the athletes participated, they had average runtime values $(127.79 \pm 4.36 \mathrm{~s}$ for the first and $142.85 \pm 5.54 \mathrm{~s}$ for the second official run) higher than those found in the literature to date. Significant correlations were found between the second official run and the second simulated race $(r=0.80)$.

Though time records are provided, studies reporting canoe slalom runtimes (Hunter et al., 2007, 2008; Zamparo et al., 2006) do not determine the individual distance travelled during the runs. Zamparo et al., (2006) studied medium to high performance athletes in Italy on a course with six gates against the current, but did not report the distance travelled during the race simulation. This parameter was investigated in this study (208.25 $\pm 11.19 \mathrm{~m}$ for the first race simulation and $216.00 \pm 4.75 \mathrm{~m}$ for the second race simulation); there was not a difference between the two simulations. On the other hand, a low and negative correlation was obtained for the distance travelled in the two simulated races. This can be attributed to differences in strategy between the two races adopted by the athletes, as the mean velocity did not differ and was significantly correlated between the two races (Table 1).

Kendal and Sanders (1992) observed that kayak velocity fluctuates during the paddle cycle. During paddling, kayak velocity increases due to the propulsive force of the blade; this force is maximised when the kayakers maximise power and minimise energy expenditure (Michael et al., 2010). During intervals between paddles, water resistance slows the kayak (Baudouin \& Hawkins, 2004; Jackson, 1995; Mann \& Kearney, 1980). A limitation of this study is that only mean velocity was measured, not velocity at each point during the paddling cycle.

Sporting events such as canoe slalom that are not covered by the media are usually recorded by athletes and coaches, with the aim of generating quantitative and qualitative information on the executed actions (Hughes \& Bartlett, 2002; Hunter et al., 2007, 2008). Among these actions, it is possible to highlight the total number of paddles and paddling characteristics.

The total number of paddles observed in both simulated competitions in this study was close to 136 . There was no difference between the two simulations $(p=0.37)$ and there was a significant correlation (ICC $=0.81)$ and a low coefficient of variation $(\mathrm{CV}=4.53)$ between the simulations, which suggests reproducibility in relation to this technical standard. In the study of the 2005 World Championship finals, Hunter et al., (2008) observed lower values for the total number of paddles (approximately 109), with runs of shorter duration than in the present investigation.

When analysing paddling characteristics within a competitive model (Total.Paddles, Pad.With, Pad.Against, Compl.cycle and Cross.Paddling), similar results were obtained for both simulations, but there were poor correlations between the first and second simulations. It is possible to speculate that among the strategies adopted by each kayaker to pass through the marked "gates", the most common was to utilise provided 
paddling (i.e., Cross.Paddling) without changing the total number of paddles. There were no studies found in the literature that analysed the paddling fractions adopted in the present investigation. Papers studying only paddling characteristics, such as the Samphao et al., (2009) study, typically use qualitative biomechanical analysis rather than quantitative analysis. Notwithstanding, during competitive events, efforts are performed at maximal intensity with pronounced participation of anaerobic alactic, lactic and anaerobic metabolism, depending on the sport and activities required to achieve victory (Gordon, Hopkins, King, Keiller \& Barnes, 2011; Lehmann, Gastmann \& Petersen, 1992; Takahashi, Wakayoshi, Hayashi, Sakaguchi \& Kitagawa, 2009; Tanskanen et al., 2009).

In addition, many sports, both individual and team-based, have varying levels of intensity during the course of competition. In canoe slalom, there are times when high intensity is required so the athlete can move at a high velocity; there are also times of lower intensity when the athlete must pass through "gates" and around natural obstacles (Nibali et al., 2011). It is necessary to note that race strategies such as pacing are not practical in this sport, though, as the total duration is too short. Competitions where pacing is appropriate have a typical range of two minutes to an hour (Lima-Silva et al., 2010). In this sense, canoe slalom competitions are performed at maximum intensity.

Among the variables able to provide relevant information on exercise intensity, aerobic/anaerobic conditioning and training effects, two variables stand out. Lactate concentration is a valuable parameter due to the ability to obtain accurate results and the sensitivity of the parameter to training (Billat, Siverent, Py, Korallsztein \& Mercier, 2003). Secondly, heart rate is frequently used to monitor individual training loads (Achten \& Jeukendrup, 2003; Lamberts, Swart, Capostagno, Noakes \& Lambert, 2010; Michael et al., 2010; Mujika, 2010) and competition characteristics, where heart rate during competition can be expressed as a percentage of Peak.HR (Borresen \& Lambert, 2009).

Our results on [Lac] before the race simulation indicate that the athletes were indeed at rest (Figure 1). The values obtained during recovery were high, as anaerobic lactic energy was used in both simulations to supply the effort. Zamparo et al., (2006) obtained average lactate concentrations values of $8.1 \pm 1.6 \mathrm{mmol} . \mathrm{L}-$ ${ }^{1}$ after $5 \mathrm{~min}$ of official competition. These values are greater than those found in this investigation at the same time ( $6.90 \pm 2.38 \mathrm{mmol}^{-\mathrm{L}^{-1}}$ for the first and $7.46 \pm 1.97 \mathrm{mmol}^{-\mathrm{L}^{-1}}$ for the second race simulation). One important precautionary measure adopted in our study is the measurement of a lactate recovery curve rather than a single point. This is important as individual differences in lactate removal can be pronounced. Additionally, blood lactate concentrations were not different between the two simulations at any of the points analysed; there were also high correlations at each point between the two simulations (ICC values ranging from 0.90 to 0.97 ), suggesting the reproducibility of this physiological variable in simulated competitions with the same natural conditions.

Regarding HR, Achten and Jeukendrup, 2003 highlight that HR is linearly correlated with exercise intensity. Simple and accurate instruments are currently able to measure and monitor this variable, providing immediate results during training sessions. These results can be used to adjust and/or quantify session intensity. Accordingly, determining HR response has been of interest in slalom kayaking. In the study of Zamparo et al., (2006), Peak.HR was determined using an incremental test and then subjects participated in

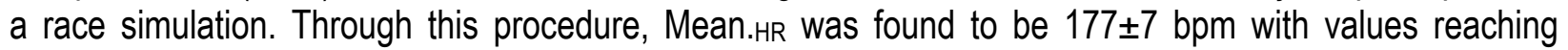
approximately $92 \%$ of Peak.HR, demonstrating the high intensity of this sport. Unlike the study conducted by Zamparo et al., (2006), we did not perform a progressive test to determine the real Peak.HR value in our paddlers; however, high HR values were obtained. Peak.HR was $183 \pm 12$ and $193 \pm 3$ bpm, Mean.HR was 
$173 \pm 14$ and $184 \pm 5 \mathrm{bpm}$, and Min.HR was $142 \pm 9$ and $151 \pm 8 \mathrm{bpm}$ for the first and second simulations, respectively. No significant correlations were found for these parameters.

\section{CONCLUSIONS}

In summary, the performance-related parameters, especially runtime and mean velocity, suggested a similarity between these simulations and official competitions. The results for these parameters were also reproducible across the two simulations. Considering the technical parameters, the total number of paddles did not change from one simulation to the next, demonstrating a high correlation between the two race simulations. On the other hand, other parameters used to describe paddling were quantitatively equal for the two simulations but were not significantly correlated. We speculated that this is attributable to different individual strategies during the two simulations. Physiological parameters of lactate concentration and heart rate suggest the intensity of the two race simulations was the same. In regard to reproducibility, key determinants which decide victory such as runtime, mean velocity and total number of paddles showed a high correlation between the simulations, indicating that the simulation model appears to be feasible and valuable for canoe slalom assessments.

\section{ACKNOWLEDGEMENTS}

We would like to thank FAPESP and CNPq for providing financial support.

\section{REFERENCES}

1. Achten, J., \& Jeukendrup, A.E. (2003). Heart rate monitoring: applications and limitations. Sports Medicine, 33(7), pp.517-538.

2. Baudouin, A., \& Hawkins, D. (2004). Investigation of biomechanical factors affecting rowing performance. Journal of Biomechanics, 37(7), pp.969-976

3. Billat, V.L., Siverent, O., Py, G., Korallsztein, J.P., \& Mercier, J. (2003). The concept of maximal lactate steady state: a bridge between biochemistry, physiology and sport science. Sports Medicine, 33(6), pp.407-26.

4. Bloomfield, J., Jonsson, G.K., Polman, R., Houlahan, K., \& O'Donoghue, P. (2005). 16. Temporal pattern analysis and its application in soccer.

5. Borresen, J., \& Lambert, M.I. (2009). The quantification of training load, the training response and the effect on performance. Sports Medicine, 39(9), pp.779-795.

6. Carling, C., Bloomfield, J., Nelsen, L., \& Reilly, T. (2008). The role of motion analysis in elite soccer: contemporary performance measurement techniques and work rate data. Sports Medicine, 38(10), pp.839-862.

7. Davies, K. (2003). Utilizing digital video for game, player, training and opposition analysis. Paper presented at the Communication to the International Conference on the Science and Practice of Rugby, Brisbane, Australia.

8. Engel, P.C., \& Jones, J.B. (1978). Causes and elimination of erratic blanks in enzymatic metabolite assays involving the use of NAD+ in alkaline hydrazine buffers: improved conditions for the assay of L-glutamate, L-lactate, and other metabolites. Anal Biochem., 88(2), pp.475-484.

9. Gordon, D., Hopkins, S., King, C., Keiller, D., \& Barnes, R.J. (2011). Incidence of the plateau at VO2max in dependent on the anaerobic capacity. International Journal of Sports Medicine, 32(1), pp.1-6. 
10. Gray, A.J., \& Jenkins, D.G. (2010). Match analysis and the physiological demands of Australian football. Sports Medicine, 40(4), pp.347-360.

11. Hill-Haas, S.V., Dawson, B.T., Coutts, A.J., \& Rowsell, G.J. (2009). Physiological responses and time-motion characteristics of various small-sided soccer games in youth players. Journal of Sports Science, 27(1), pp.1-8.

12. Hopkins, W.G., Schabort, E.J., \& Hawley, J.A. (2001). Reliability of power in physical performance tests. Sports Medicine, 31(3), pp.211-234.

13. Hughes, M.D., \& Bartlett, R.M. (2002). The use of performance indicators in performance analysis. Journal of Sports Science, 20(10), pp.739-754.

14. Hunter, A., Cochrane, J., \& Sachlikidis, A. (2007). Canoe slalom--competition analysis reliability. Sports Biomech, 6(2), pp.155-170.

15. Hunter, A., Cochrane, J., \& Sachlikidis, A. (2008). Canoe slalom competition analysis. Sports Biomech, 7(1), pp.24-37.

16. Jackson, P.S. (1995). Performance prediction for Olympic kayaks. Journal of Sports Science, 13(3), pp.239-245.

17. Kendal, S.J., \& Sanders, R.H. (1992). The technique of elite flatwater kayak paddlers using the wing paddle. International. Journal of Sports Medicine, 8(3), pp.233-250.

18. Lamberts, R.P., Swart, J., Capostagno, B., Noakes, T.D., \& Lambert, M.I. (2010). Heart rate recovery as a guide to monitor fatigue and predict change in performance parameters. Scandinavian Journal of Medicine \& Science in Sports, 20(3), pp.449-57.

19. Lehmann, M., Gastmann, U., \& Petersen, K.G. (1992). Training-over-training: performance and hormone levels, after a defined increase in training volume vs intensity in experienced middle and long-distance runners. British Journal of Sports Medicine, 26(4), pp.233-242.

20. Lima-Silva, A.E., Bertuzzi, R.C., Pires, F.O., Barros, R.V., Gagliardi, J.F., Hammond, J., Bishop, D. J. (2010). Effect of performance level on pacing strategy during a 10-km running race. European Journal of Applied Physiology, 108(5), pp.1045-1053.

21. Mann, R.V., \& Kearney, J.T. (1980). A biomechanical analysis of the Olympic-style flatwater kayak stroke. Medicine and Science Sports Exercise, 12(3), pp.183-188.

22. Martin, D., Carl, K., \& Lehnertz, K. (2008). Manual de Teoria do Treinamento Esportivo. São Paulo, IN: Phorte.

23. Matveev, L.P. (1997). Treino Desportivo: metodologia e planejamento: Phorte.

24. Messias, L.H.D., Reis, I.M., Ferrari, H.G., \& Manchado-Gobatto, F.B. (2014). Physiological, psychological and biomechanical parameters applied in canoe slalom training: a review. International Journal of Performance Analysis in Sport, 14(1), pp.24-41.

25. Michael, J.S., Rooney, K.B., \& Smith, R. (2008). The metabolic demands of kayaking: A review. Journal of Sports Science and Medicine, 7(1), pp.1-7.

26. Michael, J.S., Smith, R., \& Rooney, K. (2010). Physiological responses to kayaking with a swivel seat. International Journal of Sports Medicine, 31(8), pp.555-560.

27. Mujika, I. (2010). Intense training: the key to optimal performance before and during the taper. Scandinavian Journal of Medicine \& Science in Sports, 20(2), pp.24-31.

28. Nibali, M., Hopkins, W.G., \& Drinkwater, E. (2011). Variability and predictability of elite competitive salom-kayak performance. European Journal of Sport Science, 11(2), pp.5.

29. Samphao, S., Wheeler, A.J., Rafferty, E., Michaelson, J.S., Specht, M.C., Gadd, M.A., Smith, B.L. (2009). Diagnosis of breast cancer in women age 40 and younger: delays in diagnosis result from underuse of genetic testing and breast imaging. Am J Surg., 198(4), pp.538-543 
30. Schokman, P., Le Rossignol, P.F., \& Sparrow, W.A. (2002). Validity and reliability of a voicerecognition game analysis system for field sports. Journal of Science Medicine Sport, 5(4), pp.362371.

31. Shephard, R.J. (1987). Science and medicine of canoeing and kayaking. Sports Medicine, 4(1), pp.19-33.

32. Takahashi, S., Wakayoshi, K., Hayashi, A., Sakaguchi, Y., \& Kitagawa, K. (2009). A method for determining critical swimming velocity. International Journal of Sports Medicine, 30(2), pp.119-123.

33. Tanskanen, M., Uusitalo, A.L., Hakkinen, K., Nissila, J., Santtila, M., Westerterp, K.R., \& Kyrolainen, H. (2009). Aerobic fitness, energy balance, and body mass index are associated with training load assessed by activity energy expenditure. Scandinavian Journal Medicine Science Sports, 19(6), pp.871-878.

34. Vieira, N.A., Messias, L.H.D., Terezani, D., Borin, J.P., Cunha, S.A., Oliveira, R.M., \& ManchadoGobatto, F.B. (2011). Relationship among load training, aerobic/anaerobic parameters and performance during 22-weekes of slalom kayakers training. Paper presented at the 16th Congress of the European College of Sports science.

35. Vieira, N.A., Terezani, D., Schimdt, A., Cesar, M.C., Pellegrinotti, I.L., \& Manchado-Gobatto, F.B. (2010). Simulation race on slalom kayak: time of course, number of strokes and blood lactate. Paper presented at the 15th Congress of the European College of Sports science.

36. Zamparo, P., Tomadini, S., Didone, F., Grazzina, F., Rejc, E., \& Capelli, C. (2006). Bioenergetics of a slalom kayak (k1) competition. Int J Sports Med., 27(7), pp.546-552. 